\title{
Structural Studies of Metabolic Products of Dopamine. III.* Crystal and Molecular Structure of (-)-Adrenaline
}

\author{
A. M. ANDERSEN
}

Department of Chemistry, University of Oslo, Oslo 3, Norway

The crystal structure of (-)-adrenaline has been determined by $X$-ray methods, using 831 observed reflections collected by counter methods. The crystals are monoclinic, space group $P 2_{1}$ with $a=7.873(2), b=6.790(2), c=8.638(2) \AA$ and $\beta=98.02(2)^{\circ}$. Least-squares refinements yielded a conventional $R$-factor of 0.053 . Standard deviations in bond lengths are $0.005-0.006 \AA$ and in bond angles $0.4^{\circ}$. The adrenaline molecules were found to exist as zwitterions in the crystals. The conformation of the adrenaline molecule corresponds closely to that usually encountered among the salts of the sympa. thomimetic amines. The crystals consist of molecular double layers parallel to (100). The molecules within a layer are linked through hydrogen bonds of the types $\mathrm{N}-\mathrm{H} \cdots \mathrm{O}$ and $\mathrm{O}-\mathrm{H} \cdots \mathrm{O}$, whereas the layers are connected by van der Waals interactions.

The hormone adrenaline (epinephrine) is a sympathomimetic catecholamine acting on both $\alpha$-and $\beta$-adrenergic receptors. The natural $R(-)$-form is considerably more active than the $S(+)$-isomer. Adrenaline is easily oxidized, and its metabolic path has during the recent years been of substantial interest to research workers in various fields. ${ }^{1}$

Information concerning the molecular geom. etry and conformation pattern of the sympathomimetic amines is essential for structureactivity relationship studies. The molecular structure of the salts of several sympathomimetic amines has been determined by $X$-ray diffraction methods, ${ }^{2}$ among them (-)-adrenaline hydrogen (+)-tartrate..$^{8}$ To the author's knowledge, none of the free amines belonging to this class of compounds have yet been subjected

* The previous publication in this series: Acta Chem. Scand. B 29 (1975) 45. to X-ray investigations. This may be due to the difficulty in obtaining suitable crystals. It could therefore prove valuable to compare the molecular geometry and conformation of the free base form of $(-)$-adrenaline with the results of the structure determinations earlier mentioned.<smiles>CNCC(O)c1ccc(O)c(O)c1</smiles>

\section{EXPERIMENTAL}

Commercially obtained (-)-adrenaline was dissolved in a $1 \mathrm{M}$ nitric acid solution. At the interface between this solution and a 1 M ammonia solution, thin colourless crystal plates appeared. The infra-red spectrum of these crystals was identical to that of the original compound.

Oscillation and Weissenberg photographs indicated monoclinic symmetry. Systematically absence of reflections $0 k 0$ with $k$ odd determined the space group to be $P 2_{1}$ since the adrenaline molecule is optically active.

Unit cell parameters were obtained from a least-squares treatment of diffractometer measurements on 15 general reflections. The threedimensional intensity data were recorded on a SYNTEX $\mathbf{P} \bar{l}$ automatic diffractometer with graphite crystal monochromated $M o K \alpha$ radiation. Intensities of 956 reflections with $2 \theta<65^{\circ}$ were measured using the $\omega-2 \theta$ scan technique, the scan speed varied from 1 to $2^{\circ} \mathrm{min}^{-1}$ depending on the peak intensity. Background counts were taken at each end of the scan range, the latter being $1.4^{\circ}$. The ratio of the summarized background counting time to scan time was 0.7. 831 reflections having net intensities greater than $2 \sigma(I)$ were considered to be ob- 
Table 1. Fractional atomic coordinates and thermal parameters with estimated standard deviations for non-hydrogen atoms $\left(\times 10^{4}\right)$. The temperature factor is given by exp $-\left(B_{11} h^{2}+B_{22} k^{2}+B_{33} l^{2}+\right.$ $\left.B_{12} h k+B_{13} h l+B_{23} k l\right)$.

\begin{tabular}{|c|c|c|c|c|c|c|c|c|c|}
\hline Atom & $x$ & $y$ & $z$ & $B 11$ & $B 22$ & B33 & $B 12$ & $B 13$ & $B 23$ \\
\hline $\begin{array}{l}\text { O1 } \\
\text { O2 } \\
\text { O3 } \\
\text { N } \\
\text { C1 } \\
\text { C2 } \\
\text { C3 } \\
\text { C4 } \\
\text { C5 } \\
\text { C6 } \\
\text { C7 } \\
\text { C8 } \\
\text { C9 }\end{array}$ & $\begin{array}{l}9522(4) \\
6554(4) \\
8423(4) \\
8213(5) \\
8157(5) \\
9092(6) \\
8609(6) \\
7128(5) \\
6205(6) \\
6729(6) \\
8680(6) \\
7604(7) \\
6876(8)\end{array}$ & $\begin{array}{r}3012(8) \\
876(8) \\
1137(7) \\
5230(7) \\
2055(7) \\
2769(9) \\
2390(8) \\
1266(10) \\
504(9) \\
904(9) \\
2642(8) \\
4369(9) \\
6354(10)\end{array}$ & $\begin{array}{r}8318(3) \\
8417(3) \\
1423(3) \\
510(4) \\
4130(4) \\
5509(5) \\
6972(4) \\
7018(4) \\
5661(5) \\
4236(5) \\
2585(5) \\
1924(5) \\
-490(6)\end{array}$ & $\begin{array}{r}121(6) \\
141(8) \\
174(7) \\
115(7) \\
84(8) \\
101(8) \\
105(9) \\
116(9) \\
100(9) \\
114(9) \\
109(9) \\
124(9) \\
159(12)\end{array}$ & $\begin{array}{l}137(8) \\
288(12) \\
154(8) \\
116(9) \\
113(11) \\
118(11) \\
96(12) \\
151(12) \\
213(16) \\
184(14) \\
118(12) \\
145(12) \\
190(16)\end{array}$ & $\begin{array}{r}47(4) \\
69(5) \\
53(4) \\
60(5) \\
65(6) \\
66(6) \\
66(6) \\
64(6) \\
91(7) \\
61(6) \\
55(5) \\
68(6) \\
120(8)\end{array}$ & $\begin{array}{r}-39(12) \\
-154(16) \\
107(14) \\
28(15) \\
29(16) \\
-35(17) \\
14(17) \\
-1(20) \\
-114(20) \\
10(21) \\
45(18) \\
5(18) \\
81(24)\end{array}$ & $\begin{array}{r}9(8) \\
-20(9) \\
-6(8) \\
11(10) \\
31(11) \\
15(12) \\
26(12) \\
31(12) \\
10(13) \\
-22(11) \\
10(11) \\
78(13) \\
7(16)\end{array}$ & $\begin{array}{r}-0(9) \\
62(13) \\
-4(11) \\
7(11) \\
10(13) \\
14(15) \\
-4(13) \\
47(16) \\
12(17) \\
5(16) \\
7(13) \\
-1(14) \\
112(20)\end{array}$ \\
\hline
\end{tabular}

Table 2. Fractional atomic coordinates $\left(\times 10^{3}\right)$ with estimated standard deviations for hydrogen atoms. The isotropic thermal parameter is $B=3.2 \AA^{2}$.

\begin{tabular}{lrrr}
\hline Atom & \multicolumn{1}{l}{$y$} & \multicolumn{1}{r}{$y$} \\
\hline HC2 & $1006(7)$ & $348(8)$ & $549(5)$ \\
HC5 & $528(6)$ & $-26(8)$ & $571(5)$ \\
HC6 & $610(6)$ & $37(7)$ & $337(5)$ \\
HC7 & $994(6)$ & $306(8)$ & $272(5)$ \\
H1C8 & $757(5)$ & $553(7)$ & $265(5)$ \\
H2C8 & $641(6)$ & $396(7)$ & $169(5)$ \\
H1C9 & $736(6)$ & $693(7)$ & $-138(5)$ \\
H2C9 & $648(6)$ & $737(8)$ & $-6(5)$ \\
H3C9 & $597(6)$ & $550(7)$ & $-91(5)$ \\
H1N & $\mathbf{8 5 8}(6)$ & $430(7)$ & $-14(5)$ \\
H2N & $920(6)$ & $604(8)$ & $91(5)$ \\
HO2 & $722(6)$ & $111(9)$ & $909(5)$ \\
HO3 & $917(6)$ & $34(8)$ & $169(5)$ \\
\hline
\end{tabular}

served, the remaining 125 were excluded from the further calculations.

The intensity data were corrected for Lorentz and polarization effects. The intensities were scaled to an absolute level by methods described by Karle and Hauptman, ${ }^{4}$ and normalized structure factor magnitudes were calculated.

Atomic form factors used were those of Doyle and Turner ${ }^{5}$ for oxygen, nitrogen, and carbon atoms, and of Stewart et al. ${ }^{6}$ for hydrogen atoms.

Computer programs employed during the present study are described in Refs. 7 and 8 .

\section{CRYSTAL DATA}

Adrenaline, $\mathrm{C}_{9} \mathrm{H}_{13} \mathrm{O}_{3} \mathrm{~N}$

Space group $P 2_{1}$, monoclinic

$a=7.873(2) \AA ; b=6.790(2) \AA, c=8.638(2) \AA$; $\beta=98.02(2)^{\circ} \quad V=457.2 \AA^{3} ; M=183.22 ; F_{(000)}=$ 196; $Z=2 ; D_{\text {calc }}=1.331 \mathrm{~g} \mathrm{~cm}^{-3}$

\section{STRUCTURE DETERMINATION}

The structure was determined by use of the MULTAN program system, ${ }^{8}$ starting with $|E|$ values of 149 reflections $(|E| \geq 1.30)$. The program produced 16 sets of phases as possible solutions. An $E$ map calculated from the set of phases with the highest absolute figure of merit revealed the positions of all non-hydrogen atoms. One Fourier refinement, based on these positional parameters, yielded an $R$-factor of 0.14. Coordinates of six hydrogen atoms bonded to carbon atoms were calculated. Methyl hydrogen atoms were not included. A few cycles of least-squares refinement of positional and anisotropic thermal parameters of the non-hydrogen atoms lowered the $R$-factor to 0.078 . In three successive difference Fourier maps the seven remaining hydrogen atoms were located in positions showing a zwitterionic structure of the adrenaline molecule. The zwitterionic structure was further indicated by the infra-red spectrum of the crystals which showed a broad absorption band centered about $2500 \mathrm{~cm}^{-1}$. Subsequent

Acta Chem. Scand. B 29 (1975) No. 2 
Table 3. Bond lengths $(\AA)$ and bond angles $\left(^{\circ}\right)$ with estimated standard deviations in parantheses.

\begin{tabular}{lllll}
\hline Bond length & & Corrected & Bond angle & \\
C1-C2 & $1.397(5)$ & 1.399 & C2-C1-C6 & $118.4(4)$ \\
C2-C3 & $1.394(5)$ & 1.396 & C1-C2-C3 & $122.1(4)$ \\
C3-C4 & $1.398(6)$ & 1.405 & C2-C3-C4 & $117.4(4)$ \\
C4-C5 & $1.390(6)$ & 1.392 & C3-C4-C5 & $121.3(4)$ \\
C5-C6 & $1.379(6)$ & 1.381 & C4-C5-C6 & $119.6(5)$ \\
C1-C6 & $1.382(6)$ & 1.389 & C5-C6-C1 & $121.2(4)$ \\
C1-C7 & $1.504(6)$ & 1.507 & C6-C1-C7 & $122.3(4)$ \\
C3-O1 & $1.346(5)$ & 1.348 & C2-C1-C7 & $119.2(4)$ \\
C4-O2 & $1.374(5)$ & 1.376 & C2-C3-O1 & $123.0(4)$ \\
C7-C8 & $1.512(6)$ & & O1-C3-C4 & $119.6(4)$ \\
C8-N & $1.492(6)$ & & C3-C4-O2 & $120.5(4)$ \\
N-C9 & $1.478(6)$ & & O2-C4-C5 & $118.2(4)$ \\
C7-O3 & $1.426(5)$ & & C1-C7-O3 & $113.9(4)$ \\
C2-HC2 & $0.90(5)$ & & O3-C7-C8 & $105.8(3)$ \\
C5-HC5 & $0.90(5)$ & & C1-C7-C8 & $109.4(4)$ \\
C6-HC6 & $0.91(5)$ & & C7-C8-C & $112.7(4)$ \\
C7-HC7 & $1.02(5)$ & & & $113.4(4)$ \\
C8-H1C8 & $1.01(5)$ & & & \\
C8-H2C8 & $0.98(5)$ & & & \\
C9-H1C9 & $0.98(5)$ & & & \\
C9-H2C9 & $0.91(5)$ & & & \\
C9-H3C9 & $0.95(5)$ & & & \\
N-H1N & $0.91(5)$ & & & \\
N-H2N & $0.97(5)$ & & & \\
O2-HO2 & $0.75(5)$ & & & \\
O3-HO3 & $0.81(5)$ & & & \\
\hline
\end{tabular}

least-squares refinements including the hydrogen atoms with a common isotropic thermal parameter yielded a conventional $R$-factor of 0.053 and a weighted $R$-factor of 0.038 . A total difference Fourier map demonstrated irregular electron density with the largest peaks less than $0.3 \ominus \AA^{-8}$.

The final parameters for non-hydrogen atoms are listed in Table 1 and for hydrogen atoms in Table 2.

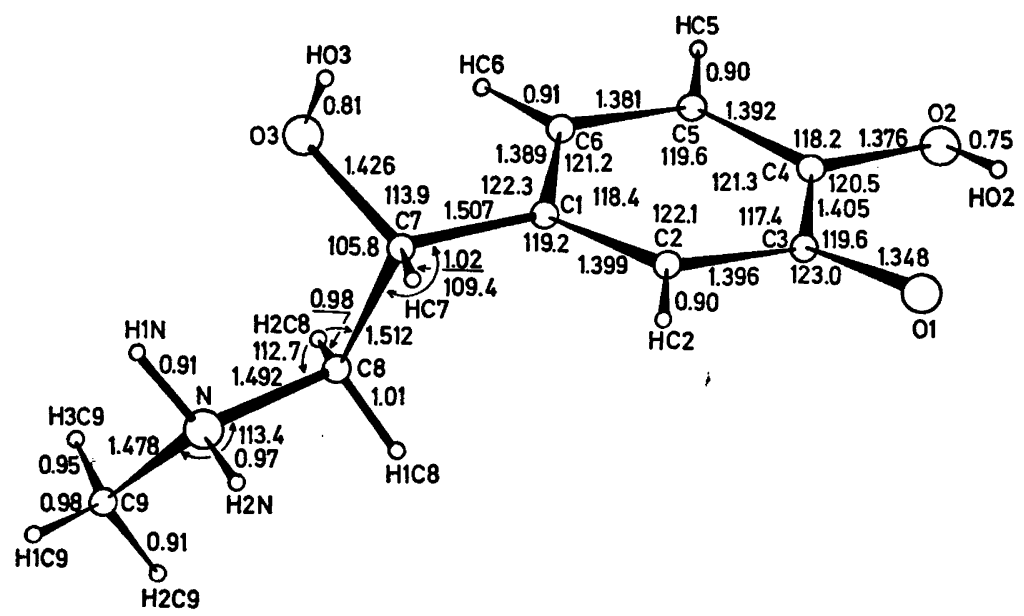

Fig. 1. Bond lengths (corrected) ( $\AA$ ) and angles ( ${ }^{\circ}$ ).

Acta Chem. Scand. B 29 (1975) No. 2 


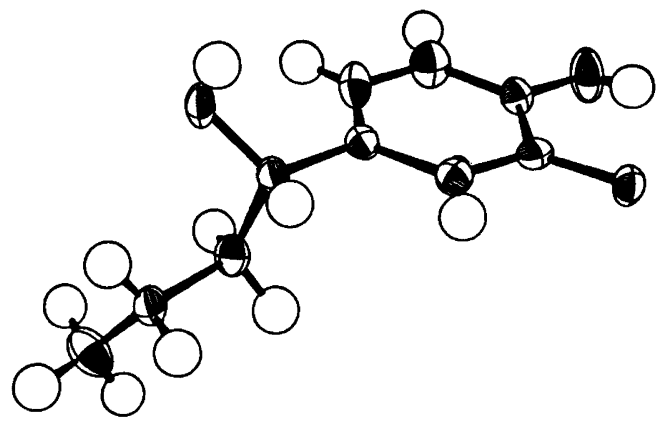

Fig. 2. The ellipsoids of thermal motion with a probability of $50 \%$. (The drawing was prepared using the computer program ORTEP ${ }^{15}$ ).

An analysis of the molecular motions in terms of rigid body vibrations indicated that the catechol part of the adrenaline molecule could be regarded as a rigid unit with the axis of the largest oscillation amplitude nearly parallel to the C1-C3 direction. The bond lengths in this part of the molecule were corrected for thermal vibration effects.

Bond lengths and angles are given in Table 3. The numbering of the atoms is indicated in Fig. 1, which also presents bond lengths (corrected) and angles. Fig. 2 shows the $R$-configuration of the adrenaline molecule including the ellipsoids of thermal motion.

The structure factor list may be obtained from this institute upon request.

\section{DISCUSSION}

Molecular structure. An interesting feature of this crystal structure is the appearance of the adrenaline molecules as zwitterions, with one proton transfered from the meta phenolic hydroxyl group to the nitrogen atom. The zwitterionic structure is indicated by the tetrahedral bonding arrangement around the nitrogen atom. The two $\mathrm{C}-\mathrm{N}$ distances ( 1.478 and $1.492 \AA$ ) are both in the range normally reported for $\mathrm{C}-\mathrm{N}+$ ingle bonds, ${ }^{2-18}$ and the two $\mathrm{N}-\mathrm{H}$ distances $(0.91$ and $0.97 \AA$ ) are in agreement with that usually found for this type of bond in $\mathrm{X}$-ray analyses. The $\mathrm{Ol}$ oxygen atom appears to be the carrier of the negative charge of the zwitterion. No hydrogen atom could be located in the vicinity of this oxygen atom, and furthermore, the C3-O1 bond length of $1.348 \AA$

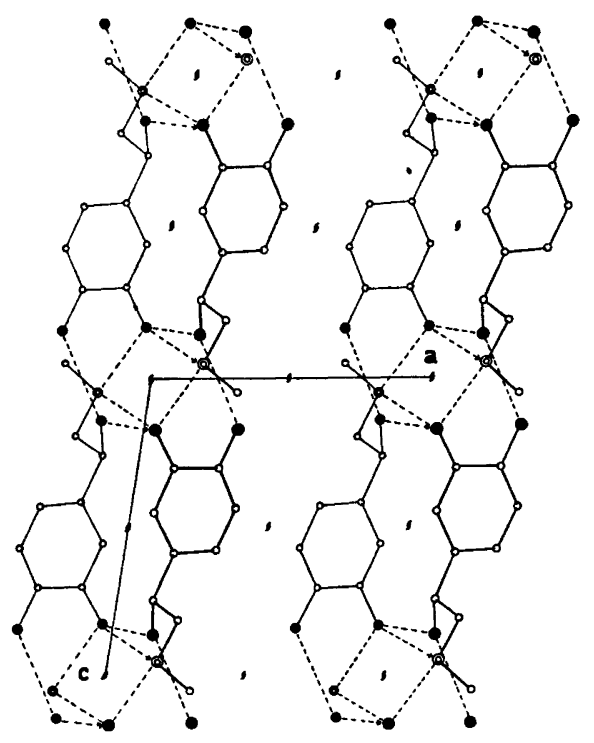

Fig. 3. The structure as viewed down the $b$-axis.

is significantly shorter than the $\mathrm{C4}-\mathrm{O} 2$ bond of $1.376 \AA$. The latter distance, as well as the O2-HO2 distance of $0.75 \AA$, represents a normal value for phenols. However, the C3-O1 separation is larger than would be expected for a $\mathrm{C}-\mathrm{O}^{-}$bond. This relative lengthening may be attributed to the formation of three fairly short hydrogen bonds having the $\mathrm{Ol}$ oxygen atom as a hydrogen acceptor (see below). The other bond lengths and bond angles are in agreement with corresponding values found in $(-)$-adrenaline

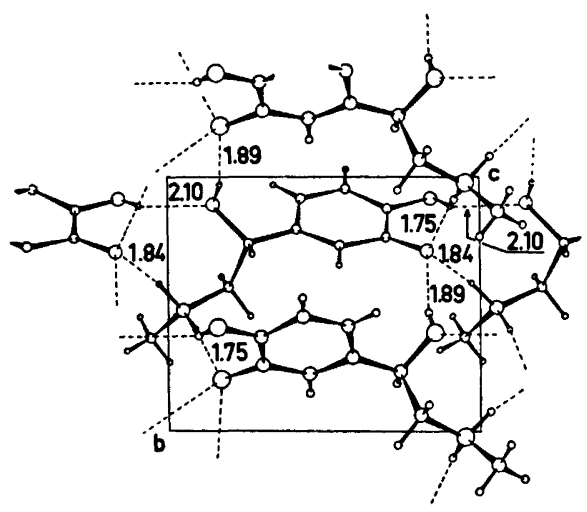

Fig. 4. The structure as viewed down the $a$-axis showing the hydrogen bonds $(\AA)$.

Acta Chem. Scand. B 29 (1975) No. 2 
Table 4. Hydrogen bonded interactions $\mathrm{X}-\mathrm{H} \cdots \mathrm{Y}$.

\begin{tabular}{|c|c|c|c|c|c|}
\hline $\mathbf{x}$ & $\mathbf{Y}$ & & $\begin{array}{l}X \cdots Y \\
(\AA)\end{array}$ & $\begin{array}{l}\mathrm{H} \cdots \mathrm{Y} \\
(\AA)\end{array}$ & $\underset{\left({ }^{\circ}\right)}{X}-H \cdots Y$ \\
\hline $\begin{array}{l}\mathrm{N} \\
\mathrm{N} \\
\mathrm{O} 3 \\
\mathrm{O} 2\end{array}$ & $\begin{array}{l}\text { O1 } \\
\text { O1 } \\
\text { O1 } \\
\text { O3 }\end{array}$ & $\begin{array}{rr}x, y, & z-1) \\
\left(-x+2, y+\frac{1}{2},\right. & -z+1) \\
\left(-x+2, y-\frac{1}{2},\right. & -z+1) \\
x \quad, y, & z+1)\end{array}$ & $\begin{array}{l}2.732 \\
2.695 \\
2.657 \\
2.806 \\
\end{array}$ & $\begin{array}{l}1.84 \\
1.75 \\
1.89 \\
2.10 \\
\end{array}$ & $\begin{array}{l}165 \\
163 \\
160 \\
157\end{array}$ \\
\hline
\end{tabular}

hydrogen $(+)$ tartrate $^{3}$ and related com. pounds. ${ }^{2-11}$

The catechol part of the adrenaline molecule is nearly planar. The deviations of the ring carbon atoms from a least-squares plane through these atoms are in the range $0.004-0.012 \AA$. The exocyclic atoms are situated $-0.065 \AA$ (O1), $0.042 \AA(\mathrm{O} 2)$ and $0.114 \AA$ (C7) out of the ring plane. Likewise, the ethylamine side chain is nearly planar, the dihedral angle $\mathrm{Cl}-\mathrm{C} 7-$ $\mathrm{C} 8-\mathrm{N}\left(\tau_{2}\right)$ is $171.6^{\circ}$. Such a fully extended side chain has always been found in the crystal structures of the salts of the sympathomimetic amines.2 The plane of the side chain of the adrenaline molecule is approximately perpendicular to the ring plane, the dihedral angle $\mathrm{C} 6-\mathrm{C} 1-\mathrm{C} 7-\mathrm{C} 8\left(\tau_{1}\right)$ is $80.5^{\circ}$. In the crystal structures of the salts of the closely related amines, the $\tau_{1}$ torsion angle is with only a few exceptions ${ }^{2,3,13}$ found to have a value about $\pm 90^{\circ} .(-)$-Adrenaline hydrogen (+) $\operatorname{tartrate}^{3}$ is one of the few related compounds with a different arrangement of the ring and side chain in the crystals, having a $\tau_{1}$ torsion angle close to $0^{\circ}$. However, the planarity of the latter molecule is assumed to be an effect of the particular molecular packing arrangement and hydrogen bond system of this crystal structure. The conformation of the adrenaline molecule in the present investigation is furthermore in agreement with the quantum mechanical calculations performed by Pullman et al. on free $N$-protonated adrenaline. ${ }^{14}$

The hydroxyl group of the side chain is found to be in a trans position relative to the meta oxygen atom of the phenyl ring. The dihedral angle $\mathrm{C} 6-\mathrm{Cl}-\mathrm{C} 7-\mathrm{O} 3$ is $-37.7^{\circ}$. In (-)-adre-

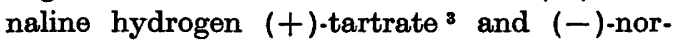
adrenaline hydrochloride, ${ }^{10}$ both with an $R$ configuration, the two hydroxyl groups are in a cis position. These latter molecules are thus reported to have a hydrophobic and a hydrophilic side in contrast to the adrenaline molecule in the present crystals.

Molecular packing and hydrogen bonding. The arrangement of the molecules in the crystals is illustrated in Fig. 3 and Fig. 4. The structure is characterized by double layers parallel to (100), containing hydrogen bonded molecules. The molecular double layers are held together by van der Waals interactions. This molecular arrangement resembles that found in the crystal structure of DL-o-tyrosine. ${ }^{12}$ The shortest intermolecular $\mathrm{C} \cdots \mathrm{C}$ and $\mathrm{C} \cdots \mathrm{O}$ contacts between the double layers in the present structure are 3.89 and 3.40 $\AA$, respectively.

The adrenaline molecules within a double layer are linked through hydrogen bonds of the types $\mathrm{N}-\mathrm{H} \cdots \mathrm{O}$ and $\mathrm{O}-\mathrm{H} \cdots \mathrm{O}$. The hydrogen bond network is arranged in infinite spirals about the $b$-axis. Hydrogen bond distances are given in Table 4 and also indicated in Fig. 4. The negatively charged oxygen atom is acting as an acceptor in three relatively short hydrogen bonds from two nitrogen atoms and one hy. droxyl oxygen atom in neighbouring molecules. The $\mathrm{N} \cdots \mathrm{O}$ separations are $2.732 \AA$ and $2.695 \AA$ and the $0 \cdots 0$ separation $2.657 \AA$. The arrangement about the charged oxygen atom is approximately tetrahedral. The phenol oxygen atom acts as hydrogen donor in a hydrogen bond with length $2.806 \AA$ to a hydroxyl oxygen atom in a neighbouring molecule.

Each adrenaline molecule is thus bonded to four other molecules within a double layer through hydrogen bonds, and to the neighbouring molecules in the adjacent double layer by van der Waals interactions.

It is a pleasure to thank Arvid Mostad and Chr. Rømming for their helpful suggestions and their interest in this study. 


\section{REFERENCES}

1. Heacock, R. A. In Katritzky, A. R. Advan. Heterocycl. Chem. 5 (1965) 205.

2. Carlström, D., Bergin, R. and Falkenberg, G. Quart. Rev. Biophys. 6 (1973) 257.

3. Carlström, D. Acta Crystallogr. $B \quad 29$ (1973) 161 .

4. Karle,J. and Hauptman, H. Acta Crystallogr. 6 (1953) 473.

5. Doyle, P. A. and Turner, P. S. Acta Crystallogr. A 24 (1968) 390.

6. Stewart, R. F., Davidson, E. R. and Simpson, W. T. J. Chem. Phys. 42 (1965) 3175.

7. Groth, P. Acta Chem. Scand. 27 (1973) 1837.

8. Germain, G., Main, P. and Woolfson, M. M. Acta Crystallogr. A 27 (1971) 368.

9. Andersen, A. M., Mostad, A. and Rømming, C. Acta Chem. Scand. 26 (1972) 2670.

10. Carlström, D. and Bergin, R. Acta Crystallogr. 23 (1967) 313.

11. Bergin, R. Acta Crystallogr. B 27 (1971) 381 .

12. Mostad, A., Rømming, C. and Tressum, L. Acta Chem. Scand. To be published.

13. Andersen, A. M., Mostad, A. and Rømming, C. Acta Chem. Scand. In press.

14. Pullman, B., Coubeils, J. L., Courrière, P. and Gervois, J. P. J. Med. Chem. 15 (1972) 17.

15. Johnson, C. K. ORTEP, Report ORNL3794, Oak Ridge National Laboratory, Oak Ridge 1965.

Received August 29, 1974. 